\title{
Image-Guided Radiotherapy Targets Macromolecules through Altering the Tumor Microenvironment
}

\author{
Oliver K. Appelbe $^{\dagger, \ddagger}$, Qingbei Zhang ${ }^{\dagger, \ddagger}$, Charles A. Pelizzari ${ }^{\S}$, Ralph R. Weichselbaum ${ }^{\dagger, \S}$, \\ and Stephen J. Kron ${ }^{\ddagger},{ }^{*}{ }^{*}$ \\ †Ludwig Center for Metastasis Research, The University of Chicago, 5758 South Maryland \\ Avenue, MC 9006, Chicago, Illinois 60637, United States \\ ‡Department of Molecular Genetics and Cellular Biology, The University of Chicago, 929 East \\ 57th Street, GCIS W519, Chicago, Illinois 60637, United States \\ §Department of Radiation and Cellular Oncology, The University of Chicago, 5758 South \\ Maryland Avenue, MC 9006, Chicago, Illinois 60637, United States
}

\begin{abstract}
Current strategies to target tumors with nanomedicines rely on passive delivery via the enhanced permeability and retention effect, leveraging the disorganized tumor microvasculature to promote macromolecule extravasation and the reduced lymphatic and venous drainage that favor retention. Nonetheless, FDA approvals and clinical use of nanomedicines have lagged, reflecting failure to display superiority over conventional formulations. Here, we have exploited image-guided Xirradiation to augment nanoparticle accumulation in tumors. A single $5 \mathrm{~Gy}$ dose of radiation, below that required to significantly delay tumor growth, can markedly enhance delivery of macromolecules and nanoparticles. The radiation effect was independent of endothelial cell integrity, suggesting a primary role for damage to microvascular pericytes and/or interstitial extracellular matrix. Significantly, radiation-guided delivery potentiated the therapeutic effects of PEGylated liposomal doxorubicin on experimental tumors. Applied to patients, these results suggest repurposing image-guided radiotherapy as a tool to guide cancer nanomedicine delivery, enhancing local control for primary tumors and metastatic disease while limiting systemic toxicity.
\end{abstract}

\section{Graphical Abstract}

*Corresponding Author: Phone: (773) 834-0250. Fax: (773) 702-3611. skron@uchicago.edu. Author Contributions

Q.Z., C.A.P., and S.J.K. initiated the project. O.K.A. and Q.Z. led experimental design, data acquisition and analysis, and manuscript preparation. C.A.P., R.R.W., and S.J.K. participated in experimental design, data analysis, and manuscript preparation.

The authors declare no competing financial interest.

Supporting Information

The Supporting Information is available free of charge on the ACS Publications website at DOI: 10.1021/acs.molpharma-ceut. $6 \mathrm{~b} 00465$.

Radiation and perivascular apoptosis; VEGFR-2 antibody depletes the tumor endothelium to the detriment of the radiation-enhanced delivery effect; imatinib mesylate treatment leads to depletion of pericytes but does not act synergistically with IR to improve radiation-enhanced accumulation; preferential retention of Doxil in irradiated areas of tumors compared to other tissues (PDF) 


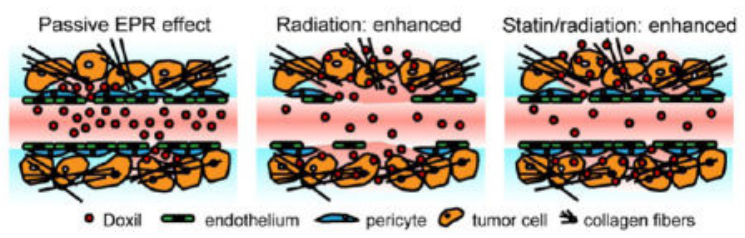

\section{Keywords}

enhanced permeability and retention; ionizing radiation; nanoparticle delivery; tumor microenvironment

\section{INTRODUCTION}

A continuing challenge for systemic cancer therapy is to focus treatment on tumors while limiting off-target toxicity. For several decades, the rationale for the development of nanoparticles as drug carriers for chemotherapy has been based upon leveraging the enhanced permeability and retention (EPR) effect, ${ }^{1}$ wherein a disorganized microvasculature is proposed to serve as an intrinsic feature characteristic of tumors that permits increased extravasation of macromolecules, while a dearth of lymphatics delays their return to circulation. The EPR effect is commonly ascribed to endothelial gaps that appear in the tortuous neovasculature of tumors. ${ }^{2,3}$ However, the EPR effect is unpredictable even in favorable models such as human xenograft tumors in nude mice and preferential delivery of macromolecules and nanoparticles to tumors in patients has been difficult to document. ${ }^{4}$ The prevalence of endothelial gaps in human tumors is uncertain, and nanoparticle eflux may be opposed by the dense microenvironment, hypoxia, and high interstitial pressure characteristic of tumors. ${ }^{5-9}$ Nanoparticle delivery may also be affected by the kinetics of transient vascular leaks ${ }^{10}$ and intermittent perfusion/cycling hypoxia. ${ }^{11,12}$ Along with a lack of clear benefit in clinical trials, nanomedicines have experienced a slow rate of FDA approvals and limited use in patients, suggesting the need to consider new approaches beyond passive delivery by the EPR effect. ${ }^{13-15}$

A number of strategies have been examined to augment the EPR effect, ${ }^{16}$ such as systemic agents that target stromal factors that may limit vascular permeability. There is also longstanding interest in the alternative strategy of image-guided drug delivery as a means to concentrate nanomedicines in tumors. ${ }^{17}$ Local heating and/or cavitation during radiofrequency ablation promotes local delivery of macromolecules and nanoparticles (e.g., refs 18,19), but these strategies are best applied to superficial tumors. Compared to ultrasound, ionizing radiation (IR) can be applied to tissue with considerably higher precision while also offering potential for deeper penetration and compatibility with complex anatomy, motion, bone and air. Classical studies of IR noted edema and plasma protein extravasation appearing within several days after a single, large X-ray dose, ${ }^{20}$ suggesting potential for image-guided drug delivery. The apparent increase in vascular permeability after IR has long been ascribed to retraction and/or destruction of microvascular endothelial cells, ${ }^{21,22}$ but other relevant contributors to enhanced extravasation and penetration may include decreased local tissue density due to cell death 
relaxing mechanical constraints and reducing particle sequestration, disruption of extracellular matrix and other stromal barriers, decreased tumor interstitial pressure, restored perfusion in hypoxic regions, vascular effects of inflammation, and other factors (e.g., refs $23,24)$. Several groups have noted the potential to exploit radiation-induced vascular permeability in guiding delivery of nanomedicines (refs 25,26 , and most recently refs $27-$ 29), but this approach has yet to demonstrate therapeutic benefit and faces the potential drawbacks of radiation exposure.

Some 50 to $60 \%$ of patients treated for cancer will receive radiotherapy during their illness, typically with curative intent. In conventional external beam radiotherapy, tumors are treated with $\sim 60-80$ Gy in daily fractions of $\sim 2-3 \mathrm{~Gy}$. Modern advances in tumor imaging and irradiation facilitate image-guided radiotherapy (IGRT) in which doses can be delivered to tumors with steep dose gradients and millimeter precision, providing minimal exposure of surrounding normal tissue. The development of IGRT has particularly advanced ablative radiotherapy (e.g., stereotactic body radiotherapy, SBRT) where cells in the targeted volume are treated with a small number of 5 to 25 Gy doses, offering a noninvasive alternative to surgical excision. Compared to conventional radiotherapy, ablative IGRT demonstrates lower adverse effects and improved local control for both primary cancers and limited metastatic disease, though the dose delivered to normal tissue remains a limiting factor. ${ }^{30}$ The larger fraction size may magnify radiation effects on both the tumor and stroma, including damage to the tumor vasculature. ${ }^{31,32}$ Only limited studies have examined combining IGRT with conventional chemotherapy or targeted agents. ${ }^{33}$ Given its expanding use and availability, we considered that repurposing IGRT as a tool to induce radiation-enhanced accumulation of therapeutics might offer a readily translatable strategy for image-guided drug delivery.

In this study, we used a small animal image-guided X-irradiation system to target nanomedicines to murine tumors, documenting delivery through multimodal intravital imaging. Utilizing a single dose of radiation to increase delivery of a liposomal chemotherapy agent, Doxil, we observed enhanced penetration of drug into the tumor parenchyma and a significantly improved treatment effect compared to radiation or Doxil on their own. The observed increase in Doxil delivery after radiation, a product of enhanced extravasation, penetration, and/or accumulation, appears to reflect favorable alterations to the tumor microenvironment that develop over several days following radiotherapy. Thus, we have established an approach to image-guided drug delivery that leverages radiation to augment macromolecule and nanoparticle delivery above that achieved via the passive EPR effect. Translating this approach may allow tumors to be treated with better-tolerated radiation doses that might be ineffective on their own, but that can efficiently target macromolecular drugs to tumors, offering improved local control and decreased risk for systemic toxicity.

\section{EXPERIMENTAL SECTION}

\section{Study Design}

Minimum sample size was calculated a priori using a power value of 0.80 . In cases where large numbers of animals would be required to obtain significance, the best judgment of the researchers regarding adequate sample size was used. Identification of outliers was 
performed using the ROUT method in Prism software. Data collection was discontinued when any dimension of a tumor reached $1-2 \mathrm{~cm}$, as stated in the University of Chicago IACUC approved ACUP\# 70931.

This research was undertaken to assess what effect varying doses of X-irradiation have on accumulation of circulating agents in tumors over time using mice to model human cancer. For tumor growth studies, treatment was initiated when tumors reached a volume of 150 $300 \mathrm{~mm}^{3}$. Animals were then assigned to treatment groups so that the mean tumor volume for each group was roughly equal. The mice were then treated with X-irradiation, injected with imaging agents at different time points, and examined using various intravital imaging modalities as detailed below. Cages contained mice from multiple treatment groups and researchers did not sort animals by treatment group when collecting data.

\section{Cell Culture}

Cell lines used in this study included the human mammary adenocarcinoma derived MCF7 ${ }^{\text {GFP-IBD }}$ (MCF7 ${ }^{\text {Tet-On }}$ (Clontech, Mountain View, CA, USA) stably transfected with doxycycline-inducible GFP fused to the 53BP1 ionizing radiation induced foci binding domain $^{34}$ ), TUBO murine mammary carcinoma (derived from BALB-neuT), and B16-F10 murine melanoma (ATCC, Manassas, VA, USA). Cells were cultured in complete growth medium (based on ATCC recommendations for each cell line) supplemented with $1 \mathrm{U} / \mathrm{mL}$ penicillin and $1 \mu \mathrm{g} / \mathrm{mL}$ streptomycin, then resuspended in sterile $1 \times$ DPBS at a concentration of $1 \times 10^{7}$ cells $/ \mathrm{mL}$ for injection of $100 \mu \mathrm{L} /$ mouse. All cell lines used in this study tested negative for mycoplasma.

\section{Mice}

Six-week-old C57BL/6, athymic nude, and BALB/c mice were purchased from Harlan Laboratories (Madison, WI, USA). One $\times 10^{6}$ in vitro cultured tumor cells (B16F10, TUBO) or $5 \times 10^{6}$ cells (MCF7 ${ }^{\mathrm{GFP}-\mathrm{IBD}}$ ) were injected subcutaneously into the hindlimb of mice to induce tumor growth. In the case of MCF7 ${ }^{\mathrm{GFP}-\mathrm{IBD}}$ tumors, athymic nude female mice received a surgical implant of an estrogen pellet $(1.7 \mathrm{mg}$ 17B-estradiol per pellet, 60 day release, Innovative Research of America, Sarasota, FL, USA) subcutaneously in their backs prior to tumor cell injection to aid in tumor growth. Female mice were used for all experiments.

Doxil (10 $\mathrm{mg} / \mathrm{kg}$ body weight) was administered via retro-orbital injection in a volume of $100 \mu \mathrm{L}$. Tumor size was measured biweekly using calipers with tumor volume calculated using the formula $V=1 w(h / 2)$. Simvastatin $(10 \mathrm{mg} / \mathrm{kg}$, Sigma-Aldrich, St. Louis, MO, USA) was administered via i.p. injection 3 days per week for 1 week prior to and after irradiation. Vascular endothelial growth factor receptor- 2 antibody $(20 \mathrm{mg} / \mathrm{kg}$, clone DC101, Bio X Cell, West Lebanon, NH, USA) was administered via i.p. injection once the day before irradiation and once more 2 days after. Imatinib mesylate $(100 \mathrm{mg} / \mathrm{kg}$, Selleck Chemicals, Houston, TX, USA) was administered via i.p. injection daily for 1 week prior to and after irradiation. All animal studies were performed in compliance and with the approval of the University of Chicago Institutional Animal Care and Use Committee, ACUP\# 70931. 


\section{Irradiation}

An X-RAD 225Cx small animal irradiator (Precision X-ray Inc., N. Bradford, CT, USA) was used to plan and deliver $\mathrm{X}$-ray ionizing radiation in a two-step process involving computed tomography ${ }^{29}$ imaging followed by image-guided delivery of a precise treatment dose. The X-ray source was used for both imaging $(1.0 \mathrm{~mm}$ focal spot) and treatment (5.5 $\mathrm{mm}$ focal spot). The subject was immobilized using isoflurane anesthesia and secured on the irradiator stage using surgical tape. Treatment planning began using the X-RAD 225C software and involved two sequential CT images taken through a $2.0 \mathrm{~mm}$ Al filter including an initial scout ( $40 \mathrm{kVp}, 0.5 \mathrm{~mA}, 0.3 \mathrm{~mm}$ isotropic voxels), followed by a more detailed full scan (40 kVp, $2.5 \mathrm{~mA}, 0.1 \mathrm{~mm}$ isotropic voxels). DICOM files of the detailed CT scan were exported into a treatment planning application written in MATLAB (The MathWorks Inc., Natick, MA, USA) for selection of the treatment isocenter, planning of an irradiation protocol, and evaluation of the selected treatment fields. In the present experiments, irradiation protocols were designed with fields from two opposing directions, each delivering half the desired total dose. The software also calculated the 3D shift of the animal support stage required to position the chosen target at the radiation isocenter for treatment. Radiotherapy was performed following import of the treatment protocol into the XRAD $225 \mathrm{C}$ software, which automated delivery of the planned fields. Treatments were performed at $225 \mathrm{kVp}, 13 \mathrm{~mA}$, and $0.3 \mathrm{~mm} \mathrm{Cu}$ filter, with either 1.5 or $2.0 \mathrm{~cm}$ diameter lead collimators providing a dose rate of $2.5 \mathrm{~Gy} / \mathrm{min}$. The irradiator output was calibrated according to the American Association of Physicists in Medicine Task Group $61^{35}$ protocol using a Farmer-type chamber. Doses ranging from 2 to 15 Gy were used in this study with total treatment time lasting up to 20 min, including setup.

\section{Intravital Imaging}

For imaging studies, mice were anesthetized by inhalation of isoflurane gas or i.p. injection of ketamine $(90 \mathrm{mg} / \mathrm{kg})$ and xylazine $(10 \mathrm{mg} / \mathrm{kg})$. AngioSense $750(100 \mu \mathrm{L})($ PerkinElmer, Waltham, MA, USA), fluorescein-labeled tomato lectin ${ }^{36}$ (50 $\mu \mathrm{L}$ in $100 \mu \mathrm{L}$ of $1 \times$ DPBS) (Vector Laboratories, Burlingame, CA, USA), and Doxil (10 mg/kg) (courtesy of the University of Chicago hospital infusion pharmacy) were administered via i.v. tail vein or retro-orbital injection. To examine fluorescence in vivo, the following instruments were utilized:

The Olympus OV100 (Olympus Corporation, Center Valley, PA, USA) is equipped with GFP, RFP, and near-infrared (NIR) fluorescence filter sets enabling multichannel coimaging. For imaging with the NIR blood pool agent AngioSense 750 and NIR iron oxide nanoparticle AngioSPARK 750 (PerkinElmer), these probes were injected via the tail vein (2 $\mathrm{nmol} /$ mouse), and fluorescence probe imaging was acquired at $1 \mathrm{~min}, 30 \mathrm{~min}, 4 \mathrm{~h}, 24 \mathrm{~h}, 3 \mathrm{~d}$, $7 \mathrm{~d}$, and $14 \mathrm{~d}$. Eight-bit planar images were acquired through GFP and the $750 \mathrm{~nm}$ channels. To study the whole body distribution of the probes, the lowest magnification lens of $0.14 \times$ was used, and the 3-10× zoom level lenses were used to study the blood vessel and microvessel leakage. Tumors were dissected and imaged using the GFP channel for fluorescein-lectin ( $\lambda_{\mathrm{EX}} 493 \mathrm{~nm}, \lambda_{\mathrm{EM}} 518 \mathrm{~nm}$ ), RFP channel for Doxil ( $\lambda_{\mathrm{EX}} 500 \mathrm{~nm}, \lambda_{\mathrm{EM}}$ $560 \mathrm{~nm}$ ), and 750 channel for AngioSense $\left(\lambda_{\mathrm{EX}} 745 \mathrm{~nm}, \lambda_{\mathrm{EM}} 800 \mathrm{~nm}\right)$ fluorescence. 
Imaging analysis was conducted using Image $(\mathrm{NIH})^{37}$ for brightness and contrast adjustment.

The Xenogen IVIS 200 (Caliper Life Sciences, Hopkinton, MA, USA) was used at $1 \mathrm{~h}, 4 \mathrm{~h}$, $24 \mathrm{~h}, 3 \mathrm{~d}, 7 \mathrm{~d}$, and $14 \mathrm{~d}$ after AngioSense injection to noninvasively image and quantitatively measure fluorescent probe permeation and retention in differentially treated tumors. An isoflurane gas anesthesia machine (XG-8 Gas Anesthesia System) was used to anesthetize mice prior to and during imaging. Dissected tumors, as well as other tissues such as liver, spleen, heart, kidney, and lung, were imaged for Doxil loading, AngioSense, and AngioSPARK fluorescence. The radiant efficiency, a relative measure of photon emission from the animal (photons $/ \mathrm{s} / \mathrm{cm}^{2} / \mathrm{sr}$ ), was measured in a standardized region of interest (ROI) with variables such as exposure time, binning, and f/stop also standardized. Intestinal Doxil retention was unable to be examined due to autofluorescence from ingested chow. ${ }^{38}$

To study the fluorescent agents at a higher magnification and resolution, a Leica SP5 Tandem Scanner Spectral 2-Photon confocal microscope (Leica Microsystems, Buffalo Grove, IL, USA) was used for live imaging. This machine provides excitation of six visible laser lines and a tunable NIR pulsed laser (Spectra Physics Mai Tai broadband 710-990 nm). Mice were anesthetized by i.p. injection of ketamine $(90 \mathrm{mg} / \mathrm{kg})$ and xylazine $(10 \mathrm{mg} / \mathrm{kg})$. Skin flap surgery was used to expose tumor tissue and blood vessels while also avoiding any autofluorescence from the skin. The exposed tumors were placed in a glass-bottom Petri dish filled with $1 \times$ PBS, and the dish was placed on the microscope stage for imaging. AngioSense, fluorescein-labeled tomato lectin, Doxil, and SAIVI nanoparticles (100 $\mu \mathrm{L}$, Invitrogen) were administered via i.v. tail vein or retro-orbital injection. Time course changes in agent fluorescence were imaged with a $20 \times$ objective lens plus digital zoom. After imaging, the tumors were dissected for other optical imaging followed by embedding for sectioning.

\section{Immunohistochemistry}

Tumors were dissected from mice immediately following sacrifice and were either fixed in $10 \%$ formalin for paraffin embedding or embedded in OCT and stored at $-80{ }^{\circ} \mathrm{C}$. Tissue processing, embedding, sectioning, and staining were performed by the Human Tissue Resource Center at the University of Chicago. Antibodies for CD31 (Santa Cruz Biotechnology, Inc., Dallas, TX, USA, sc-1506, 1:200), $a$-SMA (DAKO, Carpinteria, CA, USA, M0851, 1:100), cleaved Caspase 3 (Cell Signaling Technology, Danvers, MA, USA, \#9661, 1:200), Collagen Type IV (EMD Millipore, Billerica, MA, USA, AB8201, 1:100), and Collagen Type I (Abcam, Cambridge, MA, USA, ab34710, 1:1600) were used for IHC of tissue sections. These antibodies are routinely used throughout the literature as markers for the cell types and tissues specified herein, though some off-target signal detecting other cell types may be observed. After deparaffinization and serial rehydration, tissue sections were treated with citrate buffer pH 6.0 (S1699, DAKO) in a microwave oven. Primary antibodies were applied on tissue sections for $1 \mathrm{~h}$ at room temperature in a humidity chamber. Following a TBS wash, tissue sections were incubated with biotinylated anti-rabbit $\operatorname{IgG}(1: 200$, BA-1000, Vector laboratories) for $30 \mathrm{~min}$ at room temperature. Antigenantibody binding was detected using the VECTASTAIN Elite ABC kit (Vector Laboratories, 
PK-6100) and diaminobenzidine (DAKO, K3468) system. Tissue sections were briefly immersed in hematoxylin for counterstaining and were covered with cover glasses.

For tissue immunofluorescence, cryosections were blocked with $10 \%$ goat serum and $0.1 \%$ Triton X-100 in PBS and incubated with 1:100 primary antibody overnight at $4{ }^{\circ} \mathrm{C}$, washed, incubated with 1:400 fluorescent secondary antibodies (Invitrogen, Carlsbad CA, USA) for $30 \mathrm{~min}$, washed, and then mounted in 4',6-diamidino-2-phenyl-indole mounting medium (Vector Laboratories).

Images were captured using the Zeiss Axioskop, Zeiss Axiovert 200, and Leica SP-2 confocal microscopes. Relative quantification of staining was performed using the Color Deconvolution plugin for ImageJ to separate diaminobenzidine staining from hematoxylin. Three to five images per tumor were used for quantification. The brightness, contrast, color saturation, and color temperature of the representative images shown in Figures 2 and 3 and Supplementary Figures 1, 2, and 3 were adjusted in Microsoft Powerpoint in order to normalize the background coloring and brightness.

\section{Transmission Electron Microscopy}

Dissected tumors were fixed overnight in a $2 \%$ glutaraldehyde, $4 \%$ paraformaldehyde in 0.1 M sodium cacodylate buffer then washed with $0.1 \mathrm{M}$ sodium cacodylate buffer $3 \times$ for $5 \mathrm{~min}$ each. Buffer was then removed, and the tumor samples were incubated in $1 \%$ osmium tetroxide in $0.1 \mathrm{M}$ sodium cacodylate buffer for $60 \mathrm{~min}$, followed by two $5 \mathrm{~min}$ washes with sodium cacodylate buffer, and one 5 min wash with $\mathrm{pH} 5.1$ maleate buffer. Tumor samples were then stained in $1 \%$ uranyl acetate in maleate buffer for $60 \mathrm{~min}$, followed by three $5 \mathrm{~min}$ washes in maleate buffer and serial dehydration. Polymerization with Spurr resin was performed in an embedding oven at $60{ }^{\circ} \mathrm{C}$ for 1 to 2 days after the tumors were infiltrated by 2:1 propylene oxide/Spurr resin $2 \times$ for 30 min, then 1:1 propylene oxide/Spurr resin $2 \times$ for $30 \mathrm{~min}$, and finally $100 \%$ Spurr resin $6 \times$ for $60 \mathrm{~min}$. The blocks were trimmed and cut to 90 nm ultrathin sections using a Reichert-Jung Ultracut $E$ and mounted on 200 mesh thin bar copper grids. The specimens then were stained with uranyl acetate and lead citrate. Each specimen was examined under $300 \mathrm{kV}$ in a FEI Tecnai F30 transmission electron microscope (FEI, Hillsboro, OR, USA) with a Gatan CCD digital camera (Gatan, Pleasanton, CA, USA).

\section{Statistical Analysis}

Data are expressed as the mean \pm SEM. Statistical analyses were carried out using Prism (GraphPad Software, Inc., La Jolla, CA, USA). Prior to analyses, data to be tested were confirmed to be of a normal distribution, and F-tests were performed to compare variances between groups. In most instances, two-sided Student's $t$ test was used to determine significance with a value of $p \leq 0.05$ considered significant. For comparisons across multiple groups, two-way ANOVA was used. 


\section{RESULTS}

\section{Radiation-Enhanced Tumor Delivery of Macromolecules and Nanoparticles}

To examine radiation-induced changes in microvascular integrity after IGRT, we delivered precise doses of radiation with an image-guided $\mathrm{X}$-irradiator to flank tumors formed in athymic nude mice by subcutaneous injection of GFP-expressing MCF7 ${ }^{\text {GFP-IBD }}{ }^{\text {human }}$ breast adenocarcinoma cells. ${ }^{34}$ Serial intravital imaging of the microvasculature via fluorescence contrast ${ }^{39}$ after a single 15 Gy dose revealed minimal microscopic or macroscopic vascular disruption in the irradiated field over the first week, though significant vessel destruction was observed by 2 weeks (Figure 1A). To evaluate perfusion through the apparently intact vessels, we injected AngioSense 750EX (PerkinElmer), a near-infrared fluorescent-labeled polyethylene glycol polymer, as a blood-pool agent. Imaging during the first 2 days after 15 Gy irradiation confirmed vascular integrity. However, after 2 to 3 days, microvasculature within the irradiated field no longer retained the probe, leading to fluorescent staining of the tumor parenchyma (Figure 1B). Quantitative imaging revealed 1.2- to 3.3-fold greater accumulation of probe in tumors receiving 5 Gy versus unirradiated controls continuing up to 2 weeks after treatment (Figure 1C,D). Similar increases in tumor uptake of fluorescent probe were also observed for doses of 2 and $15 \mathrm{~Gy}$, with $15 \mathrm{~Gy}$ demonstrating greater persistence over time (Figure 1D). Notably, a single irradiation of 5 Gy is well below the dose required to decrease tumor volume or induce a significant tumor growth delay. A comparable enhancement of tumor accumulation after irradiation was observed with near-infrared fluorescent AngioSPARK 680 (PerkinElmer) PEGylated 20-35 $\mathrm{nm}$ iron-oxide nanoparticles, but this agent also displayed radiation-independent retention in lungs, liver, and spleen, consistent with uptake by the reticuloendothelial system ${ }^{40}$ (Figure 1E). To further characterize tumor penetration, we examined delivery of near-infrared fluorescent SAIVI 715 (Invitrogen) PEGylated $100 \mathrm{~nm}$ polymeric microspheres, after systemic administration. Here, confocal imaging allowed resolution of individual particles, revealing distribution throughout the parenchyma within $3 \mathrm{~h}$ of administration in the irradiated tumor volume (Figure 1F). Each agent, despite varying size and material, displayed similar enhancement after radiation.

\section{Radiation Effects on the Microenvironment Influencing Tumor Delivery}

Since the advent of fractionated radiotherapy, efforts have focused on optimizing dose and delivery to obtain maximal cytotoxic effects on tumor cells while sparing normal tissue. Nonetheless, studies have long implicated damage to tumor stroma and vasculature as critical determinants of success for therapeutic radiation. Endothelial cells serve a key role in the microvascular permeability barrier and are considered critical targets of radiation. ${ }^{22,41}$ Using fluorescein-conjugated tomato lectin to label endothelial cells, in vivo confocal imaging revealed bright, continuous staining facing the lumen of microvessels in unirradiated MCF7 GFP-IBD xenograft tumors. However, by 3 days after 5 or $15 \mathrm{~Gy}$, only weak and patchy staining of endothelium was observed (Figure 2A) concomitant with increased extravasation of AngioSense into the tumor parenchyma. Immunohistochemistry (IHC) using endothelial cell-specific CD31 antibody confirmed these results, demonstrating a significant decrease in immunoreactivity ( $p \leq 0.01$ ) after 5 or 15 Gy (Figure 2B,C). The damage to endothelial cells was not associated with increased perivascular apoptosis (Figure 
2C, Supplementary Figure 1). Pericytes are contractile cells that form a sheath around endothelial cells, controlling capillary diameter and perfusion while contributing to the regulation of permeability. ${ }^{42,43}$ Like endothelial gaps in tumor vasculature, incomplete coverage by pericytes may contribute to tumor vascular permeability and the passive EPR effect. ${ }^{44}$ IHC using alpha smooth muscle actin ( $a$-SMA) as a pericyte marker revealed a significant loss of $a$-SMA staining ( $p \leq 0.05$ ) 17 days after treatment with 2 to $15 \mathrm{~Gy}$ in $\mathrm{MCF} 7{ }^{\mathrm{GFP}-\mathrm{IBD}}$ xenograft tumors (Figure 2B,C).

To detect radiation effects on the extracellular matrix surrounding the tumor vasculature, we performed IHC for collagen types I and IV, major components of the stroma and basal lamina, respectively, that may contribute to the barrier limiting macromolecular delivery. A significant increase in collagen I staining was observed following $15 \mathrm{~Gy}$ IR, but no change was detected in the lower doses studied (Figure 2B,C). Irradiation did not significantly alter anti-collagen IV immunoreactivity either but the characteristic perivascular organization appeared disrupted (Figure 2B,C). Thin section transmission electron microscopy (TEM) at 3 days after a single 5 Gy dose demonstrated disordered alignment and packing of perivascular collagen fibers, consistent with the collagen IV IHC results (Figure 2D).

\section{Radiation-Enhanced Tumor Delivery Independent of Loss of Endothelial Integrity}

Since irradiation dose-dependent effects were observed on endothelial cells in the tumor stroma, we sought to examine whether endothelial damage might be necessary or sufficient to observe an enhanced delivery effect. Beyond their effects on cholesterol, HMG-CoA reductase inhibitors (statins) can suppress injury-induced endothelial permeability (e.g., Jacobsen et al. ${ }^{45}$ ) including radiation-induced damage. ${ }^{46,47}$ Statin effects on the microvasculature have been ascribed to reduced geranyl geranylation of Rho and/or other small GTPases. Treating mice every other day with simvastatin for a week prior to and 1 week following irradiation prevented endothelial disruption, even following $15 \mathrm{~Gy}$ irradiation (Figure 3A-C). Despite the rescue of CD31 immunoreactivity in mice treated with simvastatin and $5 \mathrm{~Gy}$, radiation still induced enhanced macromolecular delivery (Figure 3C-E).

As a complementary test for whether damaging the endothelium might be sufficient to recapitulate the effects of radiation on macromolecular delivery, we transiently stripped tumor microvasculature of endothelium by treating tumor-bearing animals with a high dose of an antibody targeting the vascular endothelial growth factor receptor-2 (VEGFR-2). ${ }^{48,49}$ When examined at $24 \mathrm{~h}$ after administration of anti-VEGFR-2, tumor microvasculature displayed significantly decreased CD31 (endothelium), $a$-SMA (pericyte), and collagen IV (basal lamina) immunoreactivity. However, the endothelial damage did not affect either passive EPR-dependent or radiation-enhanced delivery of AngioSense (Supplementary Figure 2A-C).

An alternative target of radiation affecting delivery might be pericytes. Imatinib mesylate, an inhibitor of platelet-derived growth factor receptor $\beta$ (PDGFR $\beta$ ), can deplete pericytes and disrupt tumor vascular integrity. ${ }^{50}$ Inhibition of PDGFR $\beta$-signaling by imatinib led to significant decreases in not only tumor pericyte staining but also endothelial and basal lamina immunoreactivity (Supplementary Figure 3A,B). Imatinib alone appeared to increase 
passive delivery above that due to the passive EPR effect. In combination with radiation, imatinib did not augment delivery above that displayed by radiation alone (Supplementary Figure 3C). These data are consistent with pericyte integrity and/or basal lamina as radiation targets.

Taken together, the lack of an increase in radiation-enhanced delivery following additional endothelial damage, plus the failure of simvastatin to suppress radiation-enhanced delivery despite protecting the endothelium from IR-associated damage, suggest that damage to pericytes, basement membrane, and/or extracellular matrix might have a primary role.

\section{Utilizing Image-Guided Radiation to Target Delivery of Liposomal Chemotherapy}

The apparent increase in extravasation, penetration, and accumulation of inert blood-pool agents after radiation suggested repurposing image-guided radiation as a means to enhance delivery and benefits of macromolecular and nanoparticle chemotherapies. Toward these ends, we examined the feasibility of radiation-guided delivery of the PEGylated liposomal doxorubicin agent Doxil as a model nanomedicine. ${ }^{51}$ Previous work has shown limited distribution and effectiveness of Doxil that is ascribed to its size of $\sim 100 \mathrm{~nm}$, placing it outside the ideal range for passive delivery by the EPR effect. ${ }^{52,53}$ Diverse systemic and image-guided interventions targeting the vasculature and/or extracellular matrix have been shown to enhance penetration of Doxil over that afforded by the EPR effect alone (e.g., refs 52,54-57), but results with ionizing radiation have been equivocal. ${ }^{58,59}$ As an initial test, we used the small animal image-guided irradiator to treat one-half of a MCF7 ${ }^{\mathrm{GFP}-\mathrm{IBD}}$ tumor with $15 \mathrm{~Gy}$. When $10 \mathrm{mg} / \mathrm{kg}$ Doxil was injected i.v. 3 days later, the irradiated region displayed greater doxorubicin fluorescence compared to the untreated half (Supplementary Figure 4A). Confocal microscopy after $24 \mathrm{~h}$ demonstrated the expected perivascular delivery of doxorubicin ${ }^{60}$ in the unirradiated region but nearly complete penetration into the parenchyma in the irradiated volume (Figure 4A,B).

To examine the potential therapeutic advantage provided by radiation-targeted delivery, C57BL/6 mice bearing radio-resistant B16-F10 mouse melanoma hindlimb tumors were treated with $5 \mathrm{~Gy}$ and then injected i.v. with $10 \mathrm{mg} / \mathrm{kg}$ Doxil after 3 days. Five Gy was used to remain below the radiation dose needed to cause a prolonged tumor growth delay but above the threshold for radiation-enhanced delivery. The combination significantly prolonged tumor growth delay compared to radiation or Doxil alone (Figure 4C). Notably, retention of doxorubicin was prolonged in irradiated tumors compared to other tissues (Supplementary Figure 4B).

\section{DISCUSSION}

Despite ongoing advances in conventional cancer therapies including radiotherapy, the mortality for advanced and metastatic cancers remains very high. This presents a challenge to which new approaches are sorely needed. Current methods that combine advanced imaging and computerized delivery enable ablative radiotherapy with limited risk to adjacent normal tissue, yet recurrence remains a challenge. ${ }^{30}$ Were it possible to markedly enhance cytotoxic effects within the tumor, the outcomes of image-guided radiotherapy might improve dramatically. In turn, greater efficacy might allow smaller curative doses for 
advanced local disease, both lowering toxicity and increasing the number of tumors that could be treated in the metastatic setting. Here, using preclinical models, we have characterized an increase in nanoparticle accumulation within the irradiated field, apparently mediated by dose-dependent alterations to the tumor microenvironment. Significantly, we observed that the enhanced delivery resulting from radiation requires a threshold dose of 5 Gy, with limited benefit conferred by higher doses. This observation could be due to more moderate doses of IR ( 5 Gy) allowing for increased delivery in the absence of severe vascular damage induced by larger doses ( $15 \mathrm{~Gy}$ ) that can lead to a reduction in blood perfusion and therefore delivery. ${ }^{31}$ Furthermore, the larger, 15 Gy dose led to a significant increase in collagen I immunoreactivity (Figure 2B,C), which was previously reported to reduce interstitial fluid transport and possibly restrict the movement of therapeutics within the tumor parenchyma. ${ }^{61} \mathrm{~A}$ similar increase in collagen I was not observed following treatment with the lower, 5 Gy dose (Figure 2B,C).

Toward understanding the physiological basis for enhanced delivery, we examined radiation effects on the microvasculature. As expected, endothelial damage was observed at higher radiation doses that coincided with increased tumor uptake. However, in animals treated with simvastatin, persistent endothelial integrity in tumors after irradiation did not prevent the increase in macromolecular delivery. Instead, our data implicate loss of pericyte coverage and/or perivascular collagen organization as critical determinants. Mechanistically, loss of pericytes might relax microvascular tone, increasing perfusion, while disorganization of perivascular collagen could disrupt barriers to extravasation and penetration.

Our results appear to distinguish radiation-enhanced delivery from both the EPR effect described by Maeda ${ }^{62}$ and vascular normalization as described by Jain. ${ }^{63}$ While the EPR effect is an intrinsic property of the tortuous vasculature of tumors ascribed to leakage through endothelial gaps, a relatively low dose of radiation is sufficient to promote delivery independent of endothelial integrity. Vascular normalization is a slow process that depends on revascularization as may occur following genotoxic therapy. Radiation-enhanced delivery appears to be an acute response that results directly from tissue injury, considerably prior to revascularization. Recently, Jain and colleagues have extended their normalization paradigm to the tumor microenvironment, having observed improved perfusion and drug delivery upon pharmacological targeting of extracellular matrix collagen and vascular compression. ${ }^{64}$ Distinguishing radiation-enhanced delivery is that it displays a specific radiation dose and time window and is restricted to the tumor as defined by the irradiated volume.

Tumor targeting via image-guided radiation has the potential to address a critical gap in cancer nanomedicine. While considerable progress has been made in prolonging circulation times, reducing off-target effects, and increasing uptake by tumor cells via efforts at nanoparticle engineering, a reliable and general methodology to target delivery to tumors remains lacking. Previous studies examining passive delivery of liposomal agents such as Doxil have noted limited penetration beyond perivascular tumor cells along with considerable tumor-to-tumor differences and intratumoral heterogeneity (e.g., ref 19). Using radiation to target Doxil delivery, we observed deeper penetration into the tumor parenchyma and increased drug retention after a single radiation dose. Radiation-enhanced delivery induced a tumor growth delay above that caused by radiation or Doxil alone. By 
enhancing distribution deeper into the tumor interstitium, radiation may allow Doxil access to more tumor cells, increasing the therapeutic effects without having to increase the amount administered. Furthermore, given the steep dose gradients that are readily achieved with image-guided radiation, the tissue volume exposed can be determined to nearly millimeter precision. Potentially, nanomedicine delivery can be defined to similar precision, sparing normal tissue surrounding a tumor from both the full radiation dose and the compound effects of the encapsulated chemotherapy agent.

In summary, our studies establish image-guided radiation of tumors as a practical strategy for image-guided delivery of macromolecular and nanoparticle drugs. Insofar as the target of radiation here is the stroma and microvasculature, radiation-guided delivery may demonstrate less tumor-to-tumor variability than cancer-cell targeted approaches such as conventional or ablative radiotherapy. In turn, radiation appears to augment delivery with little dependency on particle size or chemistry and may therefore enhance benefits of a wide range of nanomedicines carrying cytotoxic agents, immunotherapies, gene therapies, and/or imaging agents. Further, the low threshold radiation dose required may allow its application to anatomical sites where toxicity would otherwise prevent use of radiotherapy. Where radiation and the delivered agent display synergistic effects, it may be possible to considerably reduce radiation doses, sparing healthy tissues while focusing the cytotoxic effects on tumors. In turn, radiation targeting may allow dose reduction for systemic agents that display dose-limiting toxicities at effective doses.

\section{Supplementary Material}

Refer to Web version on PubMed Central for supplementary material.

\section{Acknowledgments}

This work was supported by the NCI via R01s CA164492 and CA199663 and by the University of Chicago Ludwig Center for Metastasis Research. We acknowledge Dr. Helena Mauceri for assistance with animal studies, Drs. ChinTu Chen and Patrick La Riviere and the staff of the Integrated Small Animal Imaging Research Resource (iSAIRR) for assistance with animal imaging, Dr. Vytas Bindokas for assistance with microscopy, Yimei Chen for performing electron microscopy, the staff of the Human Tissue Resource Center for tissue processing, embedding, and immunohistochemistry staining, and the Infusion Center pharmacy for providing PEGylated liposomal doxorubicin.

\section{References}

1. Maeda H, Nakamura H, Fang J. The EPR effect for macromolecular drug delivery to solid tumors: Improvement of tumor uptake, lowering of systemic toxicity, and distinct tumor imaging in vivo. Adv Drug Delivery Rev. 2013; 65(1):71.

2. Dvorak HF, Nagy JA, Dvorak JT, Dvorak AM. Identification and characterization of the blood vessels of solid tumors that are leaky to circulating macromolecules. American journal of pathology. 1988; 133(1):95-109. [PubMed: 2459969]

3. Hashizume H, Baluk P, Morikawa S, McLean JW, Thurston G, Roberge S, Jain RK, McDonald DM. Openings between defective endothelial cells explain tumor vessel leakiness. Am J Pathol. 2000; 156(4):1363-80. [PubMed: 10751361]

4. Wilhelm S, Tavares AJ, Dai Q, Ohta S, Audet J, Dvorak HF, Chan WCW. Analysis of nanoparticle delivery to tumours. Nature Reviews Materials. 2016; 1:16014.

5. Padera TP, Stoll BR, Tooredman JB, Capen D, di Tomaso E, Jain RK. Pathology: cancer cells compress intratumour vessels. Nature. 2004; 427(6976):695. [PubMed: 14973470] 
6. Jain RK, Baxter LT. Mechanisms of heterogeneous distribution of monoclonal antibodies and other macromolecules in tumors: significance of elevated interstitial pressure. Cancer research. 1988; 48:7022-32. [PubMed: 3191477]

7. Endrich B, Reinhold HS, Gross JF, Intaglietta M. Tissue perfusion inhomogeneity during early tumor growth in rats. Journal of the National Cancer Institute. 1979; 62(2):387-95. [PubMed: 283271]

8. Stapleton S, Milosevic M, Tannock IF, Allen C, Jaffray DA. The intratumoral relationship between microcirculation, interstitial fluid pressure and liposome accumulation. J Controlled Release. 2015; 211:163-70.

9. Ekdawi SN, Stewart JM, Dunne M, Stapleton S, Mitsakakis N, Dou YN, Jaffray DA, Allen C. Spatial and temporal mapping of heterogeneity in liposome uptake and microvascular distribution in an orthotopic tumor xenograft model. J Controlled Release. 2015; 207:101-11.

10. Matsumoto Y, Nichols JW, Toh K, Nomoto T, Cabral H, Miura Y, Christie RJ, Yamada N, Ogura T, Kano MR, Matsumura Y, Nishiyama N, Yamasoba T, Bae YH, Kataoka K. Vascular bursts enhance permeability of tumour blood vessels and improve nanoparticle delivery. Nat Nanotechnol. 2016; 11(6):533-8. [PubMed: 26878143]

11. Durand RE. Intermittent blood flow in solid tumours-an under-appreciated source of 'drug resistance'. Cancer Metastasis Rev. 2001; 20(1-2):57-61. [PubMed: 11831648]

12. Dewhirst MW, Cao Y, Moeller B. Cycling hypoxia and free radicals regulate angiogenesis and radiotherapy response. Nat Rev Cancer. 2008; 8(6):425-37. [PubMed: 18500244]

13. Venditto VJ, Szoka FC Jr. Cancer nanomedicines: so many papers and so few drugs! Adv Drug Delivery Rev. 2013; 65(1):80-8.

14. Moghimi SM, Farhangrazi ZS. Just so stories: The random acts of anti-cancer nanomedicine performance. Nanomedicine. 2014; 10:1661. [PubMed: 24832960]

15. Park K. Facing the Truth about Nanotechnology in Drug Delivery. ACS Nano. 2013; 7(9):7442. [PubMed: 24490875]

16. Prabhakar U, Maeda H, Jain R, Sevick-Muraca E, Zamboni W, Farokhzad O, Barry S, Gabizon A, Grodzinski P, Blakey D. Challenges and key considerations of the enhanced permeability and retention effect for nanomedicine drug delivery in oncology. Cancer Res. 2013; 73(8):2412. [PubMed: 23423979]

17. Lanza GM, Moonen C, Baker JR Jr, Chang E, Cheng Z, Grodzinski P, Ferrara K, Hynynen K, Kelloff G, Lee YE, Patri AK, Sept D, Schnitzer JE, Wood BJ, Zhang M, Zheng G, Farahani K. Assessing the barriers to image-guided drug delivery. Wiley interdisciplinary reviews. Nanomedicine and nanobiotechnology. 2014; 6(1):1-14. [PubMed: 24339356]

18. Monsky WL, Kruskal JB, Lukyanov AN, Girnun GD, Ahmed M, Gazelle GS, Huertas JC, Stuart KE, Torchilin VP, Goldberg SN. Radiofrequency ablation increases intratumoral liposomal doxorubicin accumulation in a rat breast tumor model. Radiology. 2002; 224(3):823-9. [PubMed: 12202721]

19. Li L, ten Hagen TL, Bolkestein M, Gasselhuber A, Yatvin J, van Rhoon GC, Eggermont AM, Haemmerich D, Koning GA. Improved intratumoral nanoparticle extravasation and penetration by mild hyperthermia. J Controlled Release. 2013; 167(2):130-7.

20. Lasnitzki I. A quantitative analysis of the direct and indirect action of $X$ radiation on malignant cells. Br J Radiol. 1947; 20(234):240-7. [PubMed: 20243691]

21. Friedman M, Ryan US, Davenport WC, Chaney EL, Strickland DL, Kwock L. Reversible alterations in cultured pulmonary artery endothelial cell monolayer morphology and albumin permeability induced by ionizing radiation. J Cell Physiol. 1986; 129(2):237-49. [PubMed: 3533961]

22. Garcia-Barros M, Paris F, Cordon-Cardo C, Lyden D, Rafii S, Haimovitz-Friedman A, Fuks Z, Kolesnick R. Tumor response to radiotherapy regulated by endothelial cell apoptosis. Science. 2003; 300(5622):1155-9. [PubMed: 12750523]

23. Multhoff G, Vaupel P. Radiation-induced changes in microcirculation and interstitial fluid pressure affecting the delivery of macromolecules and nanotherapeutics to tumors. Front Oncol. 2012; 2:165. [PubMed: 23162794] 
24. Stapleton S, Jaffray D, Milosevic M. Radiation effects on the tumor microenvironment: Implications for nanomedicine delivery. Adv Drug Delivery Rev. 2016; doi: 10.1016/j.addr. 2016.05.021

25. Lammers T, Peschke P, Kuhnlein R, Subr V, Ulbrich K, Debus J, Huber P, Hennink W, Storm G. Effect of radiotherapy and hyperthermia on the tumor accumulation of HPMA copolymer-based drug delivery systems. J Controlled Release. 2007; 117(3):333-41.

26. Lammers T, Subr V, Peschke P, Kuhnlein R, Hennink WE, Ulbrich K, Kiessling F, Heilmann M, Debus J, Huber PE, Storm G. Image-guided and passively tumour-targeted polymeric nanomedicines for radiochemotherapy. Br J Cancer. 2008; 99(6):900-10. [PubMed: 19238631]

27. Baumann BC, Kao GD, Mahmud A, Harada T, Swift J, Chapman C, Xu X, Discher DE, Dorsey JF. Enhancing the efficacy of drug-loaded nanocarriers against brain tumors by targeted radiation therapy. Oncotarget. 2013; 4(1):64-79. [PubMed: 23296073]

28. Giustini AJ, Petryk AA, Hoopes PJ. Ionizing radiation increases systemic nanoparticle tumor accumulation. Nanomedicine. 2012; 8(6):818-21. [PubMed: 22633900]

29. Moding E, Clark D, Qi Y, Li Y, Ma Y, Ghaghada K, Johnson G, Kirsch D, Badea C. Dual-energy microcomputed tomography imaging of radiation-induced vascular changes in primary mouse sarcomas. Int J Radiat Oncol, Biol Phys. 2013; 85(5):1353. [PubMed: 23122984]

30. Salama JK, Kirkpatrick JP, Yin FF. Stereotactic body radiotherapy treatment of extracranial metastases. Nat Rev Clin Oncol. 2012; 9(11):654-65. [PubMed: 23007273]

31. Park HJ, Griffin RJ, Hui S, Levitt SH, Song CW. Radiation-induced vascular damage in tumors: implications of vascular damage in ablative hypofractionated radiotherapy (SBRT and SRS). Radiat Res. 2012; 177(3):311-27. [PubMed: 22229487]

32. Hellevik T, Martinez-Zubiaurre I. Radiotherapy and the Tumor Stroma: The Importance of Dose and Fractionation. Front Oncol. 2014; 4:1. [PubMed: 24478982]

33. Zeng J, Baik C, Bhatia S, Mayr N, Rengan R. Combination of stereotactic ablative body radiation with targeted therapies. Lancet Oncol. 2014; 15(10):e426-34. [PubMed: 25186046]

34. Efimova EV, Mauceri HJ, Golden DW, Labay E, Bindokas VP, Darga TE, Chakraborty C, BarretoAndrade JC, Crawley C, Sutton HG, Kron SJ, Weichselbaum RR. Poly(ADP-ribose) polymerase inhibitor induces accelerated senescence in irradiated breast cancer cells and tumors. Cancer Res. 2010; 70(15):6277-82. [PubMed: 20610628]

35. Ma CM, Coffey CW, DeWerd LA, Liu C, Nath R, Seltzer SM, Seuntjens JP. AAPM protocol for 40-300 kV x-ray beam dosimetry in radiotherapy and radiobiology. Med Phys. 2001; 28(6):86893. [PubMed: 11439485]

36. Simionescu M, Simionescu N, Palade GE. Differentiated microdomains on the luminal surface of capillary endothelium: distribution of lectin receptors. J Cell Biol. 1982; 94(2):406-13. [PubMed: 7107706]

37. Schneider CA, Rasband WS, Eliceiri KW. NIH Image to ImageJ: 25 years of image analysis. Nat Methods. 2012; 9(7):671-5. [PubMed: 22930834]

38. Inoue Y, Izawa K, Kiryu S, Tojo A, Ohtomo K. Diet and abdominal autofluorescence detected by in vivo fluorescence imaging of living mice. Molecular imaging. 2008; 7(1):21-7. [PubMed: 18384720]

39. Zhang Q, Bindokas V, Shen J, Fan H, Hoffman RM, Xing HR. Time-course imaging of therapeutic functional tumor vascular normalization by antiangiogenic agents. Mol Cancer Ther. 2011; 10(7): 1173-84. [PubMed: 21586628]

40. Safran H, Moore T, Iannitti D, Dipetrillo T, Akerman P, Cioffi W, Harrington D, Quirk D, Rathore R, Cruff D, Vakharia J, Vora S, Savarese D, Wanebo H. Paclitaxel and concurrent radiation for locally advanced pancreatic cancer. Int J Radiat Oncol, Biol Phys. 2001; 49(5):1275-9. [PubMed: 11286834]

41. Paris F, Fuks Z, Kang A, Capodieci P, Juan G, Ehleiter D, Haimovitz-Friedman A, Cordon-Cardo C, Kolesnick R. Endothelial apoptosis as the primary lesion initiating intestinal radiation damage in mice. Science. 2001; 293(5528):293-7. [PubMed: 11452123]

42. Goddard LM, Iruela-Arispe ML. Cellular and molecular regulation of vascular permeability. Thromb Haemostasis. 2013; 109(3):407-15. [PubMed: 23389236] 
43. Shepro D, Morel NM. Pericyte physiology. FASEB journal: official publication of the Federation of American Societies for Experimental Biology. 1993; 7(11):1031-8. [PubMed: 8370472]

44. Kano M. Nanotechnology and tumor microcirculation. Adv Drug Delivery Rev. 2013; 74:2.

45. Jacobson JR, Barnard JW, Grigoryev DN, Ma SF, Tuder RM, Garcia JG. Simvastatin attenuates vascular leak and inflammation in murine inflammatory lung injury. American journal of physiology Lung cellular and molecular physiology. 2005; 288(6):L1026-32. [PubMed: 15665042]

46. Nubel T, Damrot J, Roos WP, Kaina B, Fritz G. Lovastatin protects human endothelial cells from killing by ionizing radiation without impairing induction and repair of DNA double-strand breaks. Clin Cancer Res. 2006; 12:933-9. [PubMed: 16467108]

47. Gaugler MH, Vereycken-Holler V, Squiban C, Vandamme M, Vozenin-Brotons MC, Benderitter M. Pravastatin limits endothelial activation after irradiation and decreases the resulting inflammatory and thrombotic responses. Radiat Res. 2005; 163(5):479-87. [PubMed: 15850408]

48. Gorski DH, Beckett MA, Jaskowiak NT, Calvin DP, Mauceri HJ, Salloum RM, Seetharam S, Koons A, Hari DM, Kufe DW, Weichselbaum RR. Blockage of the vascular endothelial growth factor stress response increases the antitumor effects of ionizing radiation. Cancer research. 1999; 59(14):3374-8. [PubMed: 10416597]

49. Prewett M, Huber J, Li Y, Santiago A, O’Connor W, King K, Overholser J, Hooper A, Pytowski B, Witte L, Bohlen P, Hicklin DJ. Antivascular endothelial growth factor receptor (fetal liver kinase 1) monoclonal antibody inhibits tumor angiogenesis and growth of several mouse and human tumors. Cancer research. 1999; 59(20):5209-18. [PubMed: 10537299]

50. Pietras K, Ostman A, Sjoquist M, Buchdunger E, Reed RK, Heldin CH, Rubin K. Inhibition of platelet-derived growth factor receptors reduces interstitial hypertension and increases transcapillary transport in tumors. Cancer research. 2001; 61(7):2929-34. [PubMed: 11306470]

51. Barenholz Y. Doxil®-the first FDA-approved nanodrug: lessons learned. J Controlled Release. 2012; 160(2):117.

52. Diop-Frimpong B, Chauhan VP, Krane S, Boucher Y, Jain RK. Losartan inhibits collagen I synthesis and improves the distribution and efficacy of nanotherapeutics in tumors. Proc Natl Acad Sci U S A. 2011; 108(7):2909-14. [PubMed: 21282607]

53. Harrington KJ, Rowlinson-Busza G, Syrigos KN, Uster PS, Vile RG, Peters AM, Stewart JS. The effect of irradiation on the biodistribution of radiolabeled pegylated liposomes. Int J Radiat Oncol, Biol Phys. 2001; 50(3):809-20. [PubMed: 11395251]

54. Frenkel V, Etherington A, Greene M, Quijano J, Xie J, Hunter F, Dromi S, Li KC. Delivery of liposomal doxorubicin (Doxil) in a breast cancer tumor model: investigation of potential enhancement by pulsed-high intensity focused ultrasound exposure. Academic radiology. 2006; 13(4):469-79. [PubMed: 16554227]

55. Kohli AG, Kivimae S, Tiffany MR, Szoka FC. Improving the distribution of Doxil(R) in the tumor matrix by depletion of tumor hyaluronan. J Controlled Release. 2014; 191:105-14.

56. Zheng X, Goins BA, Cameron IL, Santoyo C, Bao A, Frohlich VC, Fullerton GD. Ultrasoundguided intratumoral administration of collagenase-2 improved liposome drug accumulation in solid tumor xenografts. Cancer Chemother Pharmacol. 2011; 67(1):173-82. [PubMed: 20306263]

57. Gaber MH, Wu NZ, Hong K, Huang SK, Dewhirst MW, Papahadjopoulos D. Thermosensitive liposomes: extravasation and release of contents in tumor microvascular networks. Int J Radiat Oncol, Biol Phys. 1996; 36(5):1177-87. [PubMed: 8985041]

58. Davies, CdL; Lundstrom, LM.; Frengen, J.; Eikenes, L.; Bruland, SO.; Kaalhus, O.; Hjelstuen, MH.; Brekken, C. Radiation improves the distribution and uptake of liposomal doxorubicin (caelyx) in human osteosarcoma xenografts. Cancer Res. 2004; 64(2):547-53. [PubMed: 14744768]

59. Harrington KJ, Rowlinson-Busza G, Uster PS, Vile RG, Peters AM, Stewart JS. Single-fraction irradiation has no effect on uptake of radiolabeled pegylated liposomes in a tumor xenograft model. Int J Radiat Oncol, Biol Phys. 2001; 49(4):1141-8. [PubMed: 11240257]

60. Zhang F, Zhu L, Liu G, Hida N, Lu G, Eden HS, Niu G, Chen X. Multimodality imaging of tumor response to doxil. Theranostics. 2011; 1:302-9. [PubMed: 21772927] 
61. Znati CA, Rosenstein M, McKee TD, Brown E, Turner D, Bloomer WD, Watkins S, Jain RK, Boucher Y. Irradiation reduces interstitial fluid transport and increases the collagen content in tumors. Clinical cancer research: an official journal of the American Association for Cancer Research. 2003; 9(15):5508-13. [PubMed: 14654530]

62. Matsumura Y, Maeda H. A new concept for macromolecular therapeutics in cancer chemotherapy: mechanism of tumoritropic accumulation of proteins and the antitumor agent smancs. Cancer research. 1986; 46:6387-92. [PubMed: 2946403]

63. Jain RK. Normalization of tumor vasculature: an emerging concept in antiangiogenic therapy. Science. 2005; 307(5706):58-62. [PubMed: 15637262]

64. Liu J, Liao S, Diop-Frimpong B, Chen W, Goel S, Naxerova K, Ancukiewicz M, Boucher Y, Jain $\mathrm{R}, \mathrm{Xu}$ L. TGF- $\beta$ blockade improves the distribution and efficacy of therapeutics in breast carcinoma by normalizing the tumor stroma. Proc Natl Acad Sci U S A. 2012; 109(41):16618. [PubMed: 22996328] 

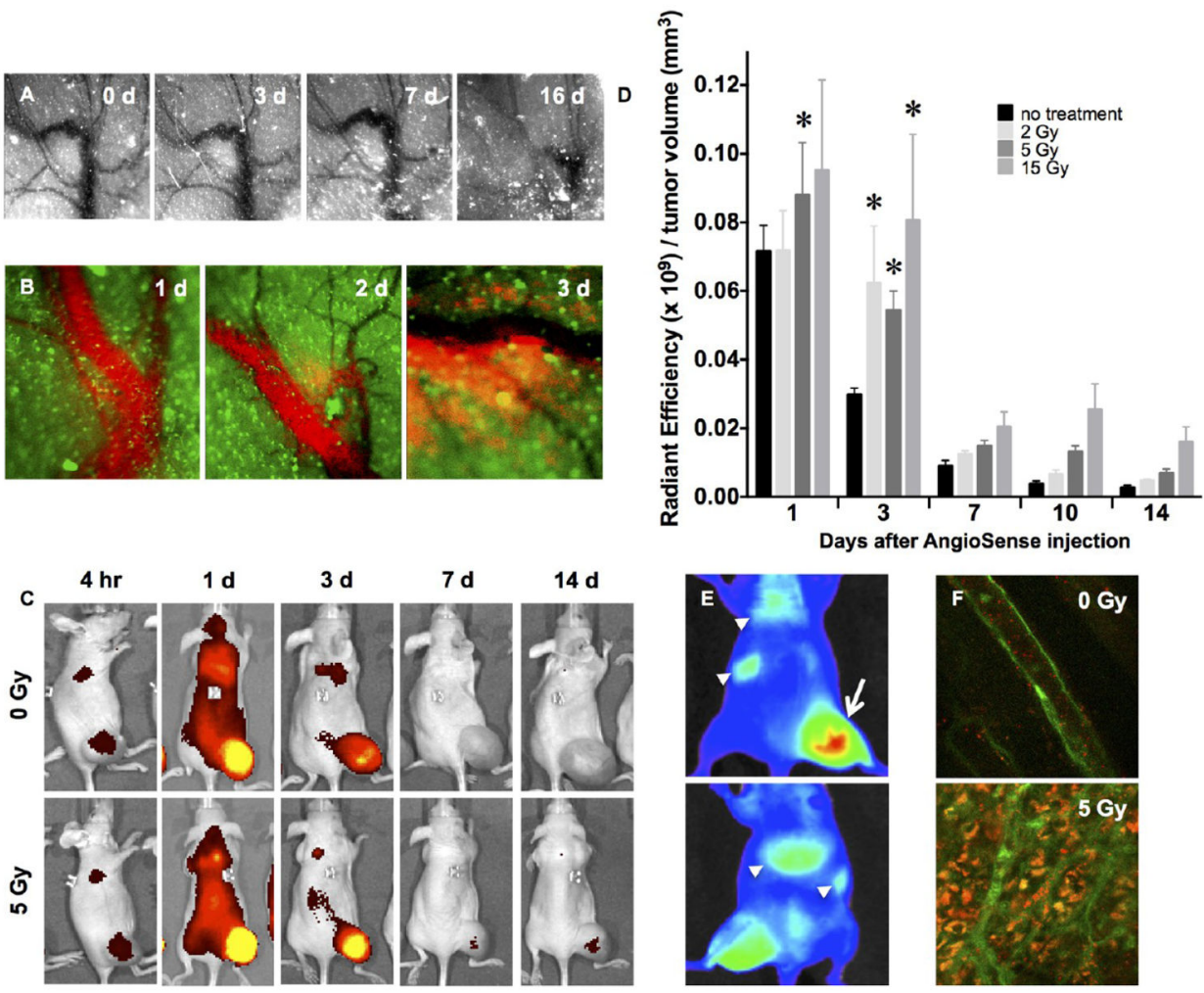

Figure 1.

Characterizing the radiation-enhanced delivery of macromolecules and nanoparticles. (A) Following 15 Gy "ablative" irradiation of a fluorescent MCF7GFP-IBD xenograft tumor, the microvasculature visualized through the skin (negative contrast) appears grossly patent through 7 days, before eventually collapsing. (B) At 3 days after 15 Gy irradiation, the macromolecular blood pool agent AngioSense 750 extravasates into MCF7GFP-IBD xenograft tumor parenchyma (green autofluorescence). (C) IVIS imaging of AngioSense 750 accumulation (black to yellow gradient) in MCF7GFP-IBD hindlimb tumors demonstrates preferential accumulation and retention after irradiation. Times listed indicate time that has passed following AngioSense administration on day 3 after irradiation. (D) Quantitation of AngioSense 750 IVIS imaging demonstrates increasing accumulation/retention with radiation dose to $\mathrm{MCF} 7^{\mathrm{GFP}-\mathrm{IBD}}$ flank tumors when probe was injected 3 days after irradiation. ${ }^{*} p \leq 0.05$ compared to control, $n=5$. (E) IVIS imaging of differential distribution (blue to red gradient) of AngioSPARK 680 PEGylated iron oxide nanoparticles after i.v. injection 3 days after 15 Gy irradiation of a MCF7GFP-IBD flank tumor (dorsal view, upper panel; ventral view, lower panel). Note enhanced accumulation in irradiated tumor (arrow) compared to lungs, spleen, and/or liver (arrowheads). (F) By $3 \mathrm{~h}$ after injection, SAIVI PEGylated $100 \mathrm{~nm}$ latex particles remained in circulation in unirradiated tumor tissue, as indicated by endothelial staining with tomato lectin (green, upper panel). By contrast, SAIVI particles spread into the parenchyma in the region where the tumor had been irradiated with 5 Gy, 3 days prior (lower panel). 


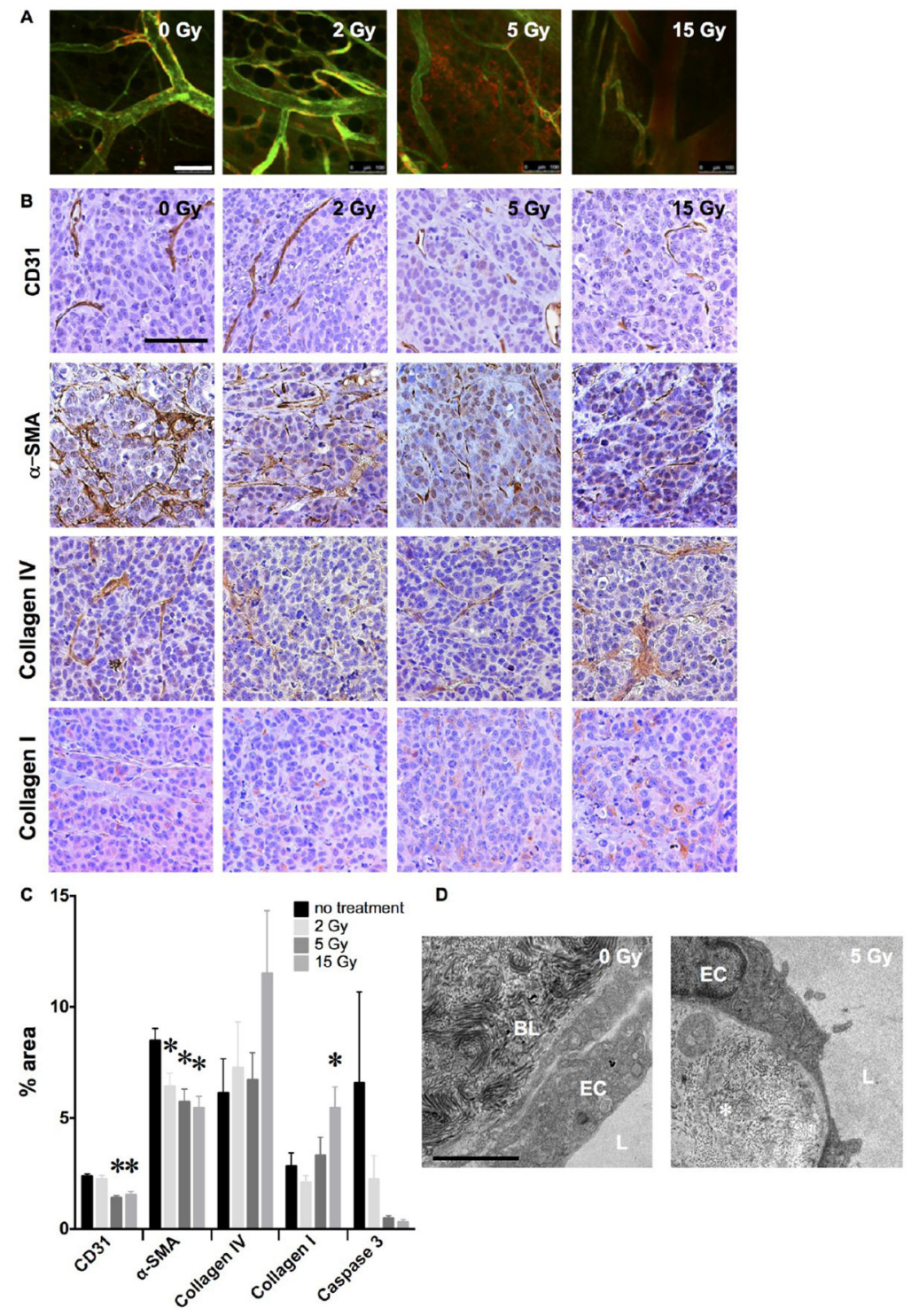

Figure 2.

Radiation alters the tumor microenvironment to enhance delivery and retention. (A) Dose response of radiation-enhanced delivery examined by confocal microscopy of MCF7GFP-IBD tumors injected with the endothelial stain tomato lectin and blood pool agent AngioSense demonstrates a threshold between 2 and 5 Gy at 3 days after irradiation. Scale bar $=100 \mu \mathrm{m}$. (B) Hematoxylin-stained (purple) tissue sections of MCF7 GFP-IBD xenograft tumors excised at 17 days after irradiation display dose-dependent changes in immunohistochemical staining (brown) for CD31 (endothelium), $a$-SMA (pericytes), Collagen IV (basement membrane), and Collagen I (extracellular matrix). Scale bar $=200 \mu \mathrm{m}$. (C) Quantification of immunohistochemical staining by percent of microscopic field. * $p \leq 0.05$ relative to 0 Gy control, $n=3$. (D) Transmission electron microscopy of thin sections of MCF7GFP-IBD xenograft tumor excised 3 days after 5 Gy demonstrates disorganization of interstitial collagen $(*)$. EC $=$ endothelial cell, $\mathrm{BL}=$ basal lamina, $\mathrm{L}=$ vessel lumen. Scale bar $=2 \mu \mathrm{m}$. 

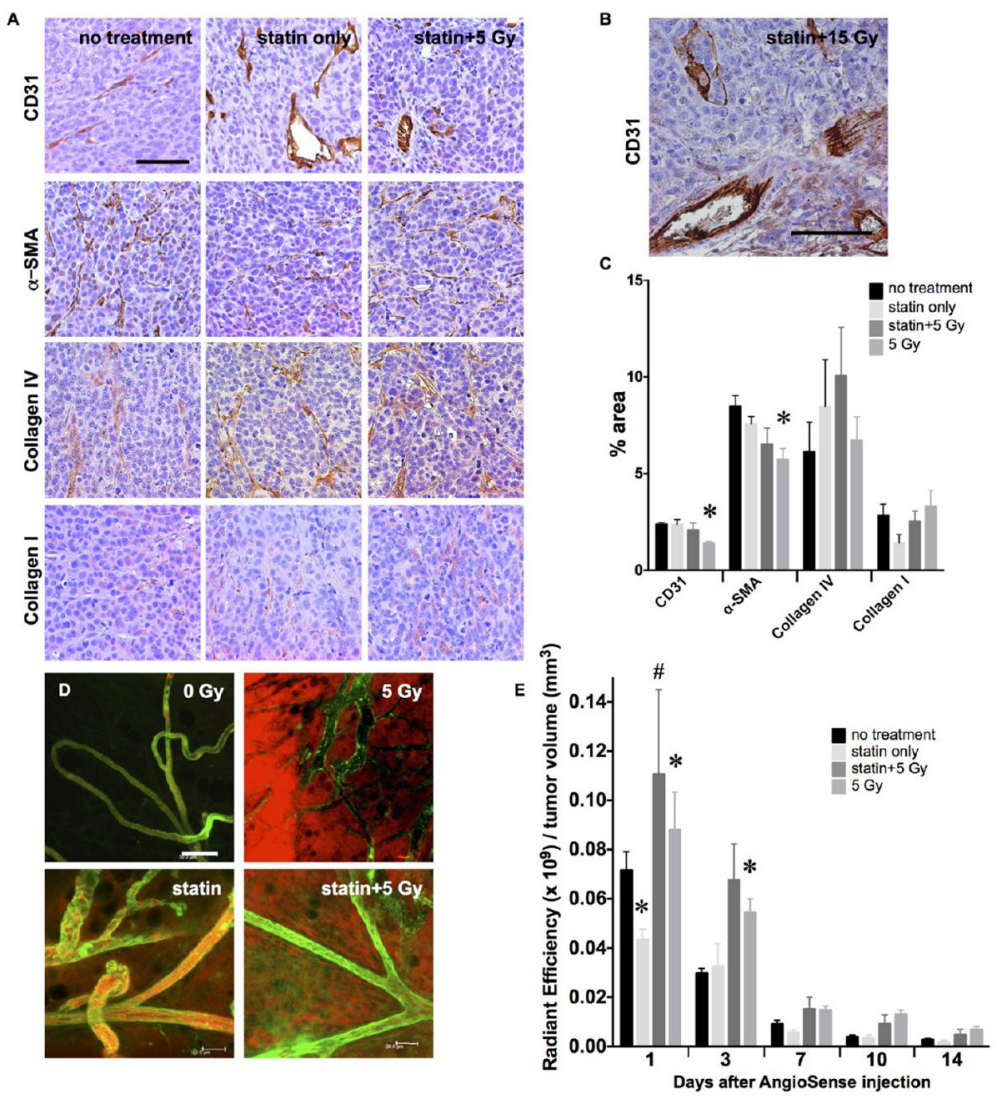

Figure 3.

Independence of radiation-enhanced delivery and endothelial integrity. (A)

Immunohistochemistry of MCF7GFP-IBD xenograft tumors excised 17 days after irradiation suggests simvastatin protects endothelial staining (brown, CD31), preventing depletion after treatment with 5 Gy. No significant effects were seen on $a$-SMA, Collagen IV, or Collagen I (brown). Purple $=$ hematoxylin, nuclei. Scale bar $=200 \mu \mathrm{m}$. (B) Simvastatin protects endothelium against destruction after $15 \mathrm{~Gy}$. (C) Relative quantification of IHC staining in MCF7GFP-IBD tumor sections. \% area denotes the area of an image stained calculated using an ImageJ macro (details in methods). ${ }^{*} p \leq 0.05$ relative to no treatment control, $n=3$. (D) In vivo imaging revealed that, despite endothelial integrity (green, tomato lectin), AngioSense still permeated from the vasculature into the MCF7GFP-IBD tumor interstitium. Scale bar $=100 \mu \mathrm{m}$. (E) MCF7GFP-IBD tumor retention of AngioSense, measured using IVIS fluorescence quantification, does not appear limited by the endothelial protection afforded by simvastatin treatment. AngioSense was administered 3 days after IR. ${ }^{*} p \leq 0.05$ relative to no treatment control; ${ }^{\#} p \leq 0.05$ relative to statin only, at the same time point, $n=5$. 

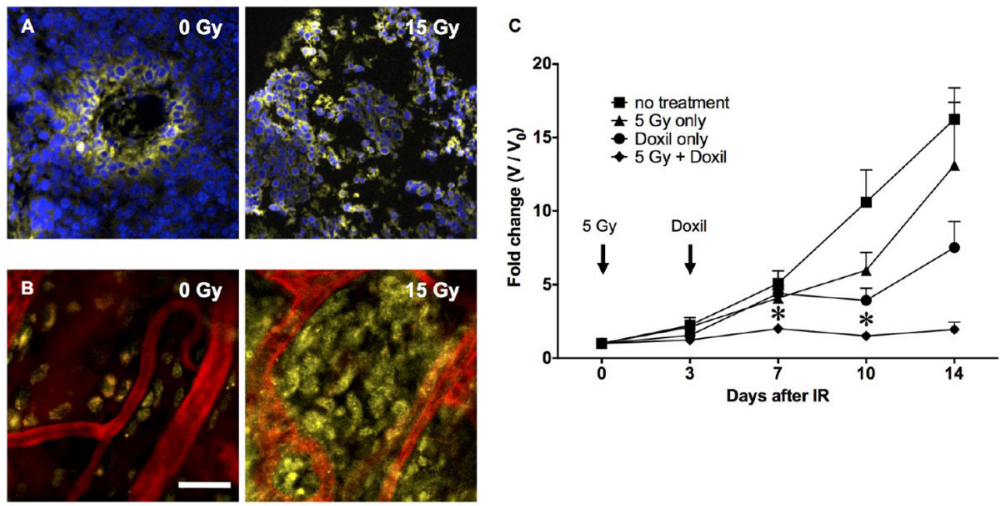

Figure 4.

Augmented delivery and therapeutic efficacy of the liposomal chemotherapy Doxil. Confocal imaging demonstrated improved homogeneous distribution of Doxil (yellow = nuclear doxorubicin) throughout irradiated MCF7 GFP-IBD xenograft tumors, using both (A) frozen sections (blue $=$ DAPI) and $(\mathrm{B})$ intravital imaging (red = AngioSense), compared to the perivascular distribution seen in controls. Scale bar $=30 \mu \mathrm{m}$. (C) C57B1/6 mice bearing syngeneic B16F10 hindlimb tumors were treated with $5 \mathrm{~Gy}$ at day 0 and/or $10 \mathrm{mg} / \mathrm{kg}$ Doxil at day 3 . Tumor growth was measured with calipers. ${ }^{*} p \leq 0.05$ compared to no treatment, radiation only, and Doxil only. $n=4$ for each data set. 